\title{
Numerical Solution of Drinfeld-Sokolov-Wilso system by using modified adomian decomposition method
}

\author{
Badran Jasim Salim', Oday Ahmed Jasim², Zeiad Yahya Ali \\ ${ }^{1,2}$ University of Mosul, College of Basic Education, Department of Mathematics, Mosul, Iraq \\ ${ }^{3}$ University of Mosul, College of physical education and sport sciences, Mosul, Iraq
}

\begin{tabular}{l} 
Article Info \\
\hline Article history: \\
Received Mar 17, 2020 \\
Revised May 14, 2021 \\
Accepted May 19, 2021 \\
\hline Keywords: \\
Modified adomian decomposition \\
method \\
Nonlinear partial differential \\
equations \\
Nonlinear System Drinfeld- \\
Sokolov-Wilso \\
Genetic algorithm
\end{tabular}

Genetic algorithm

\begin{abstract}
In this paper, the modified adomian decomposition method (MADM) is used to solve different types of differential equations, one of the numerical analysis methods for solving nonlinear partial differential equations (Drinfeld-Sokolov-Wilson system) and short (DSWS) that occur in shallow water flows. A Genetic Algorithm was used to find the optimal value for the parameter (a). We numerically solved the system (DSWS) and compared the result to the exact solution. When the value of $t$ is low and close to zero, the MADM provides an excellent approximation to the exact solution. As well as the lower value of $\mathrm{c}$ leads to the numerical algorithm of (MADM) approaching the real solution. Finally, found the optimal value when $a=-10$ by using the genetic algorithm (G-MADM). All the computations were carried out with the aid of Maple 18 and Matlab to find the parameter value (a) by using the genetic algorithm as well as to figures drawing. The errors in this paper resulted from cut errors and mean square errors.
\end{abstract}

This is an open access article under the CC BY-SA license.

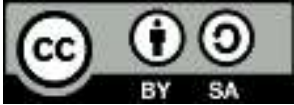

\section{Corresponding Author:}

Oday Ahmed Jasim,

Department of Mathematics,

University of Mosul,

Al-Majmooa Al-thqafiyaa Road, Mosul, Iraq.

Email: odayalnoamy@uomosul.edu.iq

\section{INTRODUCTION}

Nonlinear partial differential equations (NPDEs) are used to describe many sciences, including physics, mathematics, engineering, and chemistry, and are solved by using a number of numerical methods. George Adomian discovered and developed the method so-called a decomposition method for solving a differential equation, integro-differential, differential-delay, and partial differential equations. The solution is found as an infinite sequence that converges rapidly to accurate solutions. Nonlinear partial differential equations (NPDEs) are the best way to describe most of the engineering, mathematical, and sciences issues alike, it describes the processes of heat transfer, fluid flow, wave motion, electronic circuits as well as their use in the problems of structural structures and the mathematical description of chemical reactions [1,2].

In this paper, consider a nonlinear system (DSW), in the normalized form [3]:

$$
\left.\begin{array}{l}
\sigma_{t}-3 \rho \rho_{x}=0 \\
\rho_{t}-3 \rho_{x x x}-a\left(\sigma \rho_{x}+\rho \sigma_{x}\right)=0,
\end{array}\right]
$$

The parameter (a) is nonzero, $\sigma(x, t), \rho(x, t)$ is dependent variables, but $\mathrm{x}$, $\mathrm{t}$ independent variables. 
In the 1980's Adomian discussed a new method that provides an efficient means for the analytic and numerical solution of differential equations, and by application made to the Duffing equation found an error amount of 0.0001 [4].

A genetic algorithm (GA) is a method of optimization and research. From a Darwinian perspective, this method can be classified as one of the evolutionary algorithms that depend on imitating the work of nature. John Holland introduced genetic algorithms in 1960 based on the concept of Darwin's theory of evolution, and his student David E. Goldberg further extended GA in 1989. The genetic algorithm uses a search technique to find precise or approximate optimization solutions. Genetic algorithms are classified as Global search heuristics. Genetic algorithms are considered as one of the most important techniques in searching for the optimal choice from a set of available solutions for a specific design, and most researchers use them to find the best solution. [5]. Therefore, $t$ will be used to find the best Drinfeld-Sokolov-Wilson system.

The present paper aims at analyzing the modified decomposition method (MADM), solving the nonlinear partial differential system (DSW), monitoring the level of method accuracy in the solution and finding the optimum solution by (G_MADM). Drinfeld-Sokolov-Wilson equation used by many researchers such that; it used the F-expansion method and obtained some new exact solutions for the classic DrinfeldSokolov-Wilson equation [6]. Applied the Homotopy analysis method (HAM) to obtain the approximate solutions of Coupled Drinfeld's-Sokolov-Wilson (DSW) System and found the convergence region and the result was compared with the exact solutions [7].

Many researchers have used the ADM method to solve the following; some models of nonlinear partial differential equations [8], for linear fractional differential equations [9], nonlinear Wave-like equations with a variable coefficient [10], nonlinear integro-differential equations [11], and linear and nonlinear boundary value problems with Neumann boundary conditions [12], Burgess equation with a nonlinear source [13], a class of high order nonlinear partial differential systems (Mikhailov-Novikov-Wang and sixth-order Coupled Ramani Systems) [14].

Some researchers discovered a powerful modification of the Adomian decomposition method that will accelerate the rapid convergence of the series solution [15], a new method called the two-step Adomian decomposition method (TSADM) that will improve calculations [16], and a new Modification Adomian Decomposition Method for Nonlinear Integral Equations. [17]. However, some researchers have used the MADM method to solve the following issues: partial differential equations (Burgers equation) [18], generalized fifth-order Korteweg-de Vries (GFKdV) equation [19], for finding exact solutions of nonlinear integral equations [17], dimensional (2+1) for nonlinear system Wu-Zhang [20].

The comparison with the MADM method is as follows: Wazwaz comparison between the Variational iteration method and the adomian decomposition method [21]. Ranen compares the homotopy perturbation method to ADM; she solves some examples and illustrates the efficiency of the homotopy perturbation method [22]. Al-Amr compares Adomian's decomposition method and Variational iteration method of a Reaction-Diffusion System with fast reversible reaction [23]. Qasim applied (ADM) for nonlinear Wu Zhang system and compared the solution with MVIM, HPM, and RDTM [20]. Firoozjae compares the adomian decomposition method and differential quadrature method for solving some nonlinear partial differential equations [24].

The organization of this paper is as follows: section one provides a historical brief and introduction for MADM and ADM. Section two explains the mathematical method of the MADM. Section three displays the application and solution to the Drinfeld-Sokolov-Wilso system solves by MADM. While section four finds out the Numerical Solution for Drinfeld-Sokolov-Wilso system by MADM. Section fiveinvestigates the optimum solution by (G_MADM). Section six represents the Results and discussions of the solution by MADM, and it is more accurate and faster than other commonly used technologies. Finally, conclusion remarks are given in section seven.

\section{MATHEMATICAL OF MADM:}

Consider the system written in an operator form as:

$$
\left.\begin{array}{l}
L_{1}(\sigma)+N_{1}(\rho)+R_{1}(\rho)=\gamma_{1}(x) \\
L_{2}(\rho)+N_{2}(\sigma, \rho)+R_{2}(\sigma, \rho)=\gamma_{2}(x)
\end{array}\right\}
$$

Where $L_{1}, L_{2}$ are invertible linear operators, $N_{1}, N_{2}$ are nonlinear operators and $R_{1}, R_{2}$ linear part, from (2), getting: 


$$
\left.\begin{array}{l}
L_{1}(\sigma)=\gamma_{1}(x)-N_{1}(\rho)-R_{1}(\rho) \\
L_{2}(\rho)=\gamma_{2}(x)-N_{2}(\sigma, \rho)-R_{2}(\sigma, \rho)
\end{array}\right\}
$$

Using the initial conditions and Applied the $L^{-1}$ of (3), get:

$$
\left.\begin{array}{l}
\sigma(x, t)=\tau_{1}(x)-L_{1}^{-1} N_{1}(\rho)-L_{1}^{-1} R_{1}(\rho) \\
\rho(x, t)=\tau_{2}(x)-L_{2}^{-1} N_{2}(\sigma, \rho)-L_{2}^{-1} R_{2}(\sigma, \rho)
\end{array}\right]
$$

Where $L^{-1}$ the inverse operator, $\tau_{1}(x), \tau_{2}(x)$ represents the terms having from integrating the remaining term $\gamma_{1}(x) \cdot \gamma_{2}(x)$. ADM assumes a series the $\sigma(x, t), \rho(x, t)$ can be expressed by [25]:

$$
\begin{aligned}
& N_{1}(\rho)=\sum_{m=0}^{\infty} D_{m}\left(\rho_{o}, \rho_{1}, \ldots, \rho_{m}\right) \\
& N_{2}(\sigma, \rho)=\sum_{m=0}^{\infty} E_{m}\left(\rho_{o}, \rho_{1}, \ldots, \rho_{m}, \sigma_{o}, \sigma_{1}, \ldots, \sigma_{m}\right)
\end{aligned}
$$

Where $\mathrm{D}_{\mathrm{m}}, \mathrm{E}_{\mathrm{m}}$ are the Adomian's polynomials [4], and defined by:

$$
\left.\begin{array}{rl}
D_{m} & =\frac{1}{m !} \frac{d^{m}}{d \mu_{1} m}\left[N_{1}\left(\sum_{j=0}^{\infty} \mu_{1}^{j} \rho_{j}\right)\right]_{\mu_{1}=0^{\prime}} \\
E_{m} & =\frac{1}{m !} \frac{d^{m}}{d \mu_{2} m}\left[N_{2}\left(\sum_{j=0}^{\infty} \mu_{2}^{j} \rho_{\mathrm{j}}, \sum_{j=0}^{\infty} \mu_{2}^{j} \sigma_{\mathrm{j}}\right)\right]_{\mu_{2}=0}, \mathrm{~m}=0,1,2, \ldots \ldots ;
\end{array}\right]
$$

By Kalla polynomial can be written (5) as the following [26]:

$$
D_{m}=N_{1}\left(Z_{m}\right)-\sum_{j=0}^{m-1} D_{j}\left(\rho_{o}, \rho_{1}, \ldots, \rho_{m-1}\right)
$$

Where $Z_{m}=\rho_{o}, \rho_{1}, \ldots, \rho_{m-1}$ and $D_{\mathrm{m}}$ can be given as:

$$
\begin{aligned}
& D_{0}=N_{1}\left(\rho_{o}\right) \\
& D_{1}=\frac{d}{d x}\left(N_{1}\left(\rho_{o}\right)\right) \rho_{1}+\frac{1}{2} \frac{d^{2}}{d x^{2}}\left(N_{1}\left(\rho_{o}\right)\right) \rho_{1}^{2}+\quad \frac{1}{6} \frac{d^{3}}{d x^{3}}\left(N_{1}\left(\rho_{o}\right)\right) \rho_{1}^{3}+\frac{1}{24} \frac{d^{4}}{d x^{4}}\left(N_{1}\left(\rho_{0}\right)\right) \rho_{1}^{4}+\ldots \ldots \ldots \\
& D_{2}=\frac{d}{d x}\left(N_{1}\left(\rho_{o}\right)\right) \rho_{2}+\frac{1}{2} \frac{d^{2}}{d x^{2}}\left(N_{1}\left(\rho_{o}\right)\right)\left[2 \rho_{1} \rho_{2}+\rho_{2}^{2}\right]+\frac{1}{6} \frac{d^{3}}{d x^{3}}\left(N_{1}\left(\rho_{0}\right)\right)\left[\begin{array}{c}
3 \rho_{1}^{2} \rho_{2}+ \\
3 \rho_{1} \rho_{2}^{2}+\rho_{2}^{3}
\end{array}\right]+\ldots \ldots \ldots
\end{aligned}
$$

Similarly, find $\mathrm{E}_{\mathrm{m}}$ and $\mathrm{F}_{\mathrm{m}}$.

\section{APPLICATION MADM TO THE DSWS:}

In this section, applied the MADM to solve analytical system (1):

$$
\begin{aligned}
& L_{1(t)} \sigma-3 \rho L_{1(x)} \rho=0 \\
& L_{2(t)} \rho-3 L_{2(x x x)} \rho-a\left(L_{2(x)}(\rho) \sigma+\right. \\
& \left.L_{2(x)}(\sigma) \rho\right)=0 \\
& \text { Where, } L_{t}=\frac{\partial}{\partial_{t}}, L_{x}=\frac{\partial}{\partial_{x}}, L_{x x x}=\frac{\partial^{3}}{\partial_{x^{3}}}
\end{aligned}
$$

By applying the inverse operator $l_{t}^{-1}=\int_{0}^{t}() d$.$t then, the (7) yields:$

$$
\begin{aligned}
& \sigma(\mathrm{x}, \mathrm{t})=\gamma(\mathrm{x})+3 l_{t}^{-1}\left(\emptyset_{1}(\rho)\right), \\
& \rho(x, t)=\gamma(x)+3 L_{2(x x x)} \rho+a\left(l_{t}^{-1} \emptyset_{2}(\sigma, \rho)+l_{t}^{-1} \emptyset_{3}(\sigma, \rho)\right),
\end{aligned}
$$

Where, $\emptyset_{1}(\rho)=\rho \rho_{x}, \emptyset_{2}(\sigma, \rho)=\sigma \rho_{x}, \emptyset_{3}(\sigma, \rho)=\rho \sigma_{x}$

The MADM assumes an infinite series for $\sigma(x, t), \rho(x, t)$, as in the following:

$$
\begin{aligned}
\sigma(x, t) & =\sum_{m=0}^{\infty} \sigma_{m}(x, t) \\
\rho(x, t) & =\sum_{m=0}^{\infty} \rho_{m}(x, t)
\end{aligned}
$$


Infinite series of Adomian polynomials can be writing $\emptyset_{1}, \emptyset_{2}$, by:

$$
\emptyset_{1}(\rho)=\sum_{m=0}^{\infty} D_{m}, \emptyset_{2}(\sigma, \rho)=\sum_{m=0}^{\infty} E_{m}, \emptyset_{3}(\rho, \sigma)=\sum_{m=0}^{\infty} F_{m}
$$

Where:

$$
\left.\begin{array}{l}
D_{m}\left(\rho_{0}, \rho_{1}, \ldots \ldots \ldots, \rho_{m}\right)=\frac{1}{m !} \frac{d^{m}}{d q^{m}}\left[\vartheta_{1}\left(\sum_{k=0}^{\infty} q^{k} \rho_{k}\right)\right]_{q=0}, m \geq 0 \\
E_{m}\left(\sigma_{0}, \sigma_{1}, \ldots, \sigma_{m}, \rho_{0}, \rho_{1}, \ldots, \rho_{m}\right)=\frac{1}{m !} \frac{d^{m}}{d q^{m}}\left[\vartheta_{2}\left(\sum_{k=0}^{\infty} q^{k} \sigma_{k}, \sum_{k=0}^{\infty} q^{k} \rho_{k}\right)\right]_{q=0}, m \geq 0 \\
F_{m}\left(\rho_{0}, \rho_{1}, \ldots, \rho_{m}, \sigma_{0}, \sigma_{1}, \ldots, \sigma_{m}\right)=\frac{1}{m !} \frac{d^{m}}{d q^{m}}\left[\vartheta_{3}\left(\sum_{k=0}^{\infty} q^{k} \rho_{k}, \sum_{k=0}^{\infty} q^{k} \sigma_{k}\right)\right]_{q=0}
\end{array}\right]
$$

The first polynomials using (8), the appropriate modified Adomian polynomials, are computed by: $D_{o}=\rho_{o} \frac{\partial \rho_{o}}{\partial x}$

$$
\begin{aligned}
& D_{1}=\rho_{o} \frac{\partial \rho_{1}}{\partial x}+\rho_{1} \frac{\partial \rho_{o}}{\partial x}+\rho_{1} \frac{\partial \rho_{1}}{\partial x} \\
& D_{2}=\rho_{o} \frac{\partial \rho_{2}}{\partial x}+\rho_{1} \frac{\partial \rho_{o}}{\partial x}+\rho_{2} \frac{\partial \rho_{1}}{\partial x}+\rho_{1} \frac{\partial \rho_{2}}{\partial x}+\rho_{2} \frac{\partial \rho_{2}}{\partial x} \\
& \vdots \\
& E_{o}=\sigma_{o} \frac{\partial \rho_{o}}{\partial x} \\
& E_{1}=\sigma_{o} \frac{\partial \rho_{1}}{\partial x}+\sigma_{1} \frac{\partial \rho_{o}}{\partial x}+\sigma_{1} \frac{\partial \rho_{1}}{\partial x} \\
& E_{2}=\sigma_{o} \frac{\partial \rho_{2}}{\partial x}+\sigma_{2} \frac{\partial \rho_{o}}{\partial x}+\sigma_{2} \frac{\partial \rho_{1}}{\partial x}+\sigma_{1} \frac{\partial \rho_{2}}{\partial x}+\sigma_{2} \frac{\partial \rho_{2}}{\partial x} \\
& \vdots \\
& F_{o}=\rho_{o} \frac{\partial \sigma_{o}}{\partial x} \\
& F_{1}=\rho_{o} \frac{\partial \sigma_{1}}{\partial x}+\rho_{1} \frac{\partial \sigma_{o}}{\partial x}+\rho_{1} \frac{\partial \sigma_{1}}{\partial x} \\
& F_{2}=\rho_{o} \frac{\partial \sigma_{2}}{\partial x}+\rho_{2} \frac{\partial \sigma_{o}}{\partial x}+\rho_{2} \frac{\partial \sigma_{1}}{\partial x}+\rho_{1} \frac{\partial \sigma_{2}}{\partial x}+\rho_{2} \frac{\partial \sigma_{2}}{\partial x} \\
& \vdots
\end{aligned}
$$

And so on, by (7), writing:

$$
\begin{aligned}
& \sigma_{0}(x, t)=\rho_{0}(x, t)=\gamma(x) \\
& \sigma_{m+1}(x, t)=l_{t}^{-1}\left[3 D_{m}\right] ; \quad m \geq 1, \\
& \rho_{m+1}(x, t)=l_{t}^{-1}\left[3 L_{2(x x x)} \rho+a E_{m}+a F_{m}\right] ; m \geq 1,
\end{aligned}
$$

\section{NUMERICAL SOLUTION}

It considers the solutions of (9) with the initial and conditions [3]:

$$
\left.\begin{array}{l}
\sigma_{o}(x, t)=\frac{2 c}{\sqrt{3 a}} \operatorname{sech}\left(\sqrt{\frac{c}{3}}(x)\right) \\
\rho_{o}(x, t)=\frac{2 c}{a} \operatorname{sech}^{2}\left(\sqrt{\frac{c}{3}}(x)\right)
\end{array}\right\}
$$

To calculate the MADN for $\sigma(x, t)$ and $\rho(x, t)$, we substituted the initial conditions (10) into (9) and by using Maple 18 language got as follows:

$$
\begin{aligned}
\sigma_{1}(\mathrm{x}, \mathrm{t}) & =\frac{4}{3} \frac{\mathrm{c}^{5 / 2} \sqrt{3} \sinh (1 / 3 \sqrt{3 c} \mathrm{x})}{\mathrm{a} \cosh (1 / 3 \sqrt{3 c} \mathrm{x})^{3}} \\
\rho_{1}(x, t) & =\frac{64}{12} \frac{c^{5 / 2} t \sqrt{3} \sinh (1 / 3 \sqrt{3 c} x)}{a \cosh (1 / 3 \sqrt{3 c} x)^{3}}-\frac{64}{3} \frac{c^{5 / 2} t \sqrt{3} \sinh (1 / 3 \sqrt{3 c} x)}{a \cosh (1 / 3 \sqrt{3 c} x)^{5}}+\frac{16}{3} \frac{c^{5 / 2} t \sinh (1 / 3 \sqrt{3 c} x)}{a^{3 / 2} \cosh (1 / 3 \sqrt{3 c} x)^{4}}
\end{aligned}
$$

Now, find $\sigma_{2}$ : 


$$
\begin{gathered}
\sigma_{2}(x, t)=\frac{32}{3} \frac{c^{11 / 2} \sqrt{3} \sinh (1 / 3 \sqrt{3 c} x)}{a^{2} \cosh (1 / 3 \sqrt{3 c} x)^{5}}+\frac{8 c^{4} \sqrt{3}}{a^{3 / 2} \cosh (1 / 3 \sqrt{3 c} x)^{3}}-16 \frac{c^{11 / 2} \sqrt{3} \sinh (1 / 3 \sqrt{3 c} x)}{a^{2} \cosh (1 / 3 \sqrt{3 c} x)^{7}} \\
-\frac{32}{3} \frac{8 c^{4} \sqrt{3}}{a^{3 / 2} \cosh (1 / 3 \sqrt{3 c} x)^{5}} \\
\rho_{2}(x, t)=\frac{2}{9} \frac{1}{a^{5 / 2} \cosh \left(1 / 3^{\sqrt{3 c}} x\right)^{9}}\left[t c^{4}\left[96 a^{3 / 2} \cosh (1 / 3 \sqrt{3 c} x)^{5} t+\ldots \ldots \ldots .7560 a^{3 / 2} t \cosh (1 / 3 \sqrt{3 c} x)\right]\right]
\end{gathered}
$$

To find $\sigma$ then:

$$
\left.\begin{array}{rl}
\sigma(\mathrm{x}, \mathrm{t})= & \sigma_{\mathrm{o}}(\mathrm{x}, \mathrm{t})+\sigma_{1}(\mathrm{x}, \mathrm{t})+\sigma_{2}(\mathrm{x}, \mathrm{t})+\cdots \\
& \sigma(x, t)=\frac{2}{3} \frac{1}{a^{5 / 2} \cosh \left(\frac{1}{3} \sqrt{3 c} x\right)^{7}}\left\{\sqrt{3} c\left\{-2 \cosh \left(\frac{1}{3} \sqrt{3 c} x\right)^{4} \sinh \left(\frac{1}{3} \sqrt{3 c} x\right) a^{3 / 2} c^{3 / 2}\right]\right. \\
& -16 \cosh \left(\frac{1}{3} \sqrt{3 c} x\right)^{2} \sinh \left(\frac{1}{3} \sqrt{3 c} x\right) \sqrt{a} c^{9 / 2}+\cosh \left(\frac{1}{3} \sqrt{3 c} x\right)^{6} a^{2}+ \\
& \left.12 \cosh \left(\frac{1}{3} \sqrt{3 c} x\right)^{4} a c^{3}+24 \sinh \left(\frac{1}{3} \sqrt{3 c} x\right)^{6} \sqrt{a} c^{9 / 2}-16 \cosh \left(\frac{1}{3} \sqrt{3 c} x\right)^{2} a c^{3}\right\}
\end{array}\right\}
$$

And to find $\rho$ then:

$$
\begin{aligned}
\rho(\mathrm{x}, \mathrm{t})= & \rho_{\mathrm{o}}(\mathrm{x}, \mathrm{t})+\rho_{1}(\mathrm{x}, \mathrm{t})+\rho_{2}(\mathrm{x}, \mathrm{t})+\cdots \\
\rho(\mathrm{x}, \mathrm{t})= & \frac{2}{9} \frac{1}{\mathrm{a}^{5 / 2} \cosh \left(\frac{1}{3} \sqrt{3 \mathrm{c} x}\right)^{9}}\left\{\mathrm { c } \left\{96 \cosh \left(\frac{1}{3} \sqrt{3 \mathrm{c} x}\right)^{7} \mathrm{t}^{2} \mathrm{a}^{3 / 2} \mathrm{c}^{3}+64 \cosh \left(\frac{1}{3} \sqrt{3 c \mathrm{x}}\right)^{4}\right.\right. \\
& * \sinh \left(\frac{1}{3} \sqrt{3 \mathrm{c} x}\right) \sqrt{3 \mathrm{a}} \mathrm{c}^{9 / 2} \mathrm{t}^{2}-24 \cosh \left(\frac{1}{3} \sqrt{3 \mathrm{c} x}\right)^{6} * \sinh \left(\frac{1}{3} \sqrt{3 c} \mathrm{x}\right)^{6} \mathrm{ta}^{3 / 2} \mathrm{c}^{3 / 2}+ \\
& \left.\left.105 * \cosh \left(\frac{1}{3} \sqrt{3 c} x\right)^{6} \sqrt{3} a t^{2} c^{3}-3024 \cosh \left(\frac{1}{3} \sqrt{3 c} x\right)^{5} a^{3 / 2} t^{2} c^{3} \ldots \ldots \ldots \ldots\right\}\right\}
\end{aligned}
$$

It has got the solutions of the system (1) numerically as follows:

$$
\begin{aligned}
& \sigma(x, t)=\sum_{i=0}^{\infty} \sigma_{i}(x, t) \\
& \rho(x, t)=\sum_{i=0}^{\infty} \rho_{i}(x, t)
\end{aligned}
$$

This solution is convergent to the exact solution [27]:

$$
\begin{aligned}
& \sigma(x, t)=\frac{2 c}{\sqrt{3 a}} \operatorname{sech}\left(\sqrt{\frac{c}{3}}(x-c t)\right), \\
& \rho(x, t)=\frac{2 c}{a} \operatorname{sech}^{2}\left(\sqrt{\frac{c}{3}}(x-c t)\right),
\end{aligned}
$$

\section{EXPLAINED (G_MADM)}

Using the genetic algorithm and the MADM, this method aims to find the best nonlinear DSW parameters. The following equations are used with the MADM series solution for (11), (12):

$$
\begin{aligned}
& \sigma(a)=\sum_{i=1}^{n} \sum_{j=1}^{m}\left(\sigma\left(x_{i}, t_{j}\right)-\hat{\sigma}\left(x_{i}, t_{j}\right)\right)^{2} \\
& \rho(a)=\sum_{i=1}^{n} \sum_{j=1}^{m}\left(\rho\left(x_{i}, t_{j}\right)-\hat{\rho}\left(x_{i}, t_{j}\right)\right)^{2} \\
& S=1 / 2|\sigma(a)+\rho(a)|
\end{aligned}
$$

$\sigma$ and $\rho$ are the solutions of the system DSW for (11), (12). $\widehat{\sigma}$ and $\hat{\rho}$ are the exact solutions for the system DSW. S represents the mean square error. Thus, by using the genetic algorithm, we find that $a=-10$ is the optimum value for the systems (11), (12). This value is obtained by using the Matlab program and the genetic algorithm method, which provides the best values of the parameters in the DSW system. The errors in this paper are resulted from cut errors and mean square errors. 


\section{RESULTS AND DISCUSSION}

Comparison the absolute errors for the MADN results and exact solution for $\sigma(x, t)$ and $\rho(x, t)$, when $\mathrm{a}=0.1, \mathrm{c}=0.0001$, and $\mathrm{a}=0.1, \mathrm{c}=0.01, \mathrm{t}=1$. The lower value of $\mathrm{c}$, the closer results are to the real solution, noticed that the accuracy of the solution was excellent, as shown in (see Table 1, 2) and also (see Figure 1-4).

The solution for (1), with the initial conditions (10), by using genetic algorithm (G_MADM), we take $\mathrm{a}=-10, \mathrm{c}=0.0001, \mathrm{t}=1$, where the received value is the best value that is $\mathrm{a}=-10$ by using $\mathrm{G}$ MADM, as shown in (see Table 3 ) and also in (see Figure 5, 6).

Through the tables (see Table 1,3), we note that the results for $\rho, \sigma$ when $\mathrm{a}=0.1, \mathrm{c}=0.01, \mathrm{t}=1$ and $\mathrm{a}=-10, \mathrm{c}=0.01, \mathrm{t}=1$ by using MADM and G-MADM, respectively. We note that the results by using the genetic algorithm are better (more accurate).

By compared the absolute error between MADM and G-MADM methods, the technique (GMADM) is the best (see Table 4). The Maple 18 software was used to do all of the measurements, and the Matlab program was used to execute them.

Table 1. MADN and exact solution when $\mathrm{a}=0.1, \mathrm{c}=0.0001, \mathrm{t}=1$

\begin{tabular}{|c|c|c|c|c|c|c|}
\hline$x$ & $\sigma_{\text {MADM }}$ & $\sigma_{\text {exact }}$ & $\left|\sigma_{\text {exact }}-\sigma_{\text {MADM }}\right|$ & $\rho_{\text {MADM }}$ & $\rho_{\text {exact }}$ & $\left|\rho_{\text {exact }}-\rho_{\text {MADM }}\right|$ \\
\hline-100 & 0.0003117325775 & 0.0003117172202 & $1.535731237 * 10^{-8}$ & 0.001457666135 & 0.001457514381 & $1.517536499 * 10^{-8}$ \\
\hline-90 & 0.0003208493473 & 0.0003208348803 & $1.446696319 * 10^{-8}$ & 0.001544172364 & 0.001544025307 & $1.470569996 * 10^{-8}$ \\
\hline-80 & 0.0003293841001 & 0.0003293706933 & $1.340681763 * 10^{-8}$ & 0.001627415628 & 0.001627275804 & $1.398238919 * 10^{-8}$ \\
\hline-60 & 0.0003372301854 & 0.0003372180071 & $1.217831272 * 10^{-8}$ & 0.001705869723 & 0.001705739765 & $1.299580908 * 10^{-8}$ \\
\hline-70 & 0.0003442832529 & 0.0003442724645 & $1.078835673 * 10^{-8}$ & 0.001777970411 & 0.001777852948 & $1.174634934 * 10^{-8}$ \\
\hline-50 & 0.0003504442227 & 0.0003504349742 & $9.248549282 * 10^{-9}$ & 0.001842172509 & 0.001842070067 & $1.024415783 * 10^{-8}$ \\
\hline-40 & 0.0003556223749 & 0.0003556148007 & $7.574234942 * 10^{-9}$ & 0.001897013390 & 0.001896928298 & $8.509213832 * 10^{-7}$ \\
\hline-30 & 0.0003597384015 & 0.0003597326151 & $5.786400348 * 10^{-9}$ & 0.001941179046 & 0.001941113317 & $6.572871728 * 10^{-7}$ \\
\hline-20 & 0.0003627272382 & 0.0003627233286 & $3.909620839 * 10^{-9}$ & 0.001973567963 & 0.001973523197 & $4.476611928 * 10^{-7}$ \\
\hline-10 & 0.0003645405023 & 0.0003645385310 & $1.971257584 * 10^{-9}$ & 0.001993347787 & 0.001993325109 & $2.267820866 * 10^{-7}$ \\
\hline 0 & 0.0003651483715 & 0.0003651483698 & $1.7 * 10^{-12}$ & 0.001999999999 & 0.001999999980 & $1.9 * 10^{-11}$ \\
\hline 10 & 0.0003645407677 & 0.0003645427356 & $1.967857584 * 10^{-9}$ & 0.001993348449 & 0.001993371092 & $2.264320866^{*} 10^{-8}$ \\
\hline 20 & 0.0003627277622 & 0.0003627316683 & $3.906120839 * 10^{-9}$ & 0.001973569221 & 0.001973613949 & $4.472811928 * 10^{-8}$ \\
\hline 30 & 0.0003597391703 & 0.0003597449537 & $5.783400348 * 10^{-9}$ & 0.001941180776 & 0.001941246476 & $6.569971728 * 10^{-8}$ \\
\hline 40 & 0.0003556233691 & 0.0003556309403 & $7.571234942 * 10^{-9}$ & 0.001897015426 & 0.001897100486 & $8.506013832 * 10^{-8}$ \\
\hline 50 & 0.0003504454179 & 0.0003504546632 & $9.245349282 * 10^{-9}$ & 0.001842174655 & 0.001842277065 & $1.024095783 * 10^{-7}$ \\
\hline 60 & 0.0003442846209 & 0.0003442954068 & $1.078585673 * 10^{-8}$ & 0.001777972469 & 0.001778089907 & $1.174384934 * 10^{-7}$ \\
\hline 70 & 0.0003372316962 & 0.0003372438720 & $1.217581272 * 10^{-8}$ & 0.001705871501 & 0.001706001439 & $1.299380908 * 10^{-7}$ \\
\hline 80 & 0.0003293857223 & 0.0003293991268 & $1.340451763 * 10^{-8}$ & 0.001627416964 & 0.001627556772 & $1.398078919 * 10^{-7}$ \\
\hline 90 & 0.0003208510497 & 0.0003208655152 & $1.446546319 * 10^{-8}$ & 0.001544173134 & 0.001544320184 & $1.470499996 * 10^{-7}$ \\
\hline 100 & 0.0003117343305 & 0.0003117496864 & $1.535591237 * 10^{-8}$ & 0.001457666257 & 0.001457818005 & $1.517476499 * 10^{-7}$ \\
\hline
\end{tabular}

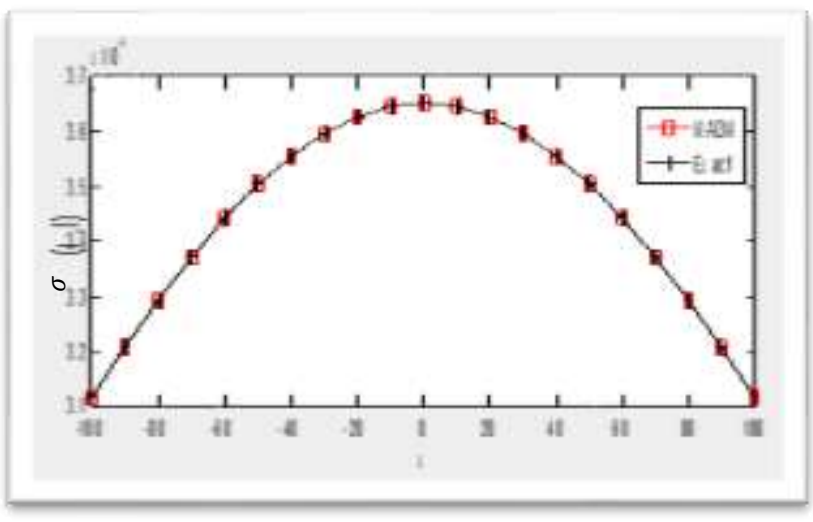

Figure 1. The MADM and Exact Solution $\sigma(\mathrm{x}, \mathrm{t})$ when $\mathrm{a}=0.1, \mathrm{t}=1, \mathrm{c}=0.0001$

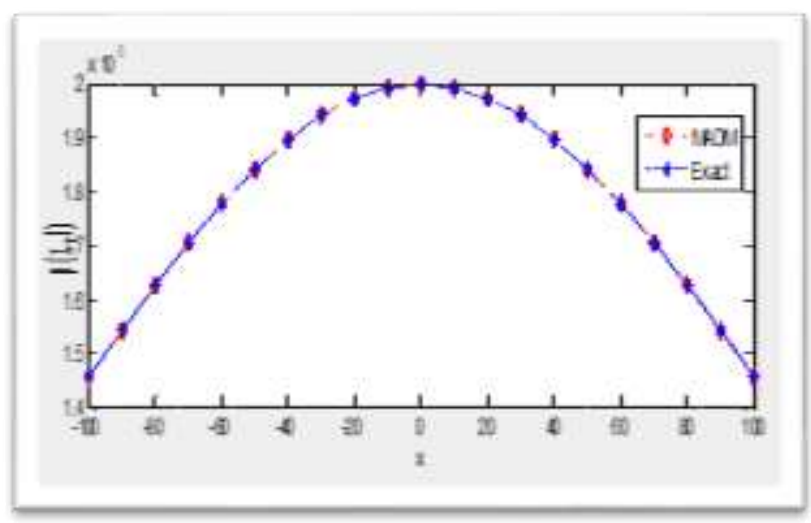

Figure 2. The MADM and Exact Solution $\mathrm{p}(\mathrm{x}, \mathrm{t})$ when $\mathrm{a}=0.1, \mathrm{t}=1, \mathrm{c}=0.0001$

Table 2. MADN and exact solation when $\mathrm{a}=0.1, \mathrm{c}=0.01, \mathrm{t}=1$ 


\begin{tabular}{|c|c|c|c|c|c|c|}
\hline $\mathrm{X}$ & $\sigma_{M A D M}$ & $\sigma_{\text {exact }}$ & $\left|\sigma_{\text {exact }}-\sigma_{\text {MADM }}\right|$ & $\rho_{M A D M}$ & $\rho_{\text {exact }}$ & $\left|\rho_{\text {exact }}-\rho_{M A D M}\right|$ \\
\hline-100 & 0.0002270271149 & 0.0002247770382 & 0.000002250076654 & 0.000007767900950 & 0.000007578707538 & $1.891934119 * 10-7$ \\
\hline-90 & 0.0004043852509 & 0.0004003898320 & 0.000003995418888 & 0.00002464780908 & 0.00002404680264 & $6.010064357 * 10-7$ \\
\hline-80 & 0.0007202489902 & 0.0007131726966 & 0.000007076293648 & 0.00007820283362 & 0.00007629229430 & $1.910539318 * 10-6$ \\
\hline-60 & 0.001282592355 & 0.001270119968 & 0.00001247238662 & 0.0002480592222 & 0.0002419807100 & $6.078512205 * 10-6$ \\
\hline-70 & 0.002282772198 & 0.002260993555 & 0.00002177864266 & 0.0007861570288 & 0.0007668137786 & $1.934325017 * 10-5$ \\
\hline-50 & 0.004056469301 & 0.004019155694 & 0.00003731360742 & 0.002484371182 & 0.002423041874 & $6.132930840 * 10-5$ \\
\hline-40 & 0.007173777969 & 0.007112415751 & 0.00006136221771 & 0.007778740525 & 0.007587968676 & $1.907718494 * 10-4$ \\
\hline-30 & 0.01250295995 & 0.01241114673 & 0.00009181322371 & 0.02365779664 & 0.02310548450 & $5.523121422 * 10-4$ \\
\hline-20 & 0.02087431859 & 0.02076488745 & 0.0001094311360 & 0.06594506369 & 0.06467708262 & $1.267981071 * 10-3$ \\
\hline-10 & 0.03108577406 & & 0.00007546400375 & & & $1.516827962 * 10-3$ \\
\hline 0 & 0.03651337657 & 0.03651301149 & $3.6508 * 10-7$ & 0.1999982308 & 0.1999800013 & $1.82295 * 10-5$ \\
\hline 10 & 0.03126107064 & 0.03133495402 & 0.00007388338375 & 0.1457749946 & 0.1472819016 & $1.506906962 * 10-3$ \\
\hline 20 & 0.02099872189 & 0.02110794232 & 0.0001092204260 & 0.06554753385 & 0.06683178436 & $1.284250511 * 10-3$ \\
\hline 30 & 0.01255403219 & 0.01264650486 & 0.00009247267371 & 0.02342967888 & 0.02399011280 & $5.604339222 * 10-4$ \\
\hline 40 & 0.007191299637 & 0.007253261278 & 0.00006196164071 & 0.007697911975 & 0.007891469878 & $1.935579034 * 10-4$ \\
\hline 50 & 0.004062142043 & 0.004099839707 & 0.00003769766442 & 0.002459067422 & 0.002521302844 & $6.223542240 * 10-5$ \\
\hline 60 & 0.002284575246 & 0.002306578340 & 0.00002200309366 & 0.0007784118300 & 0.0007980455460 & $1.963371597 * 10-5$ \\
\hline 70 & 0.001283162115 & 0.001295762093 & 0.00001259997762 & 0.0002456789788 & 0.0002518499104 & $6.170931605 * 10-6$ \\
\hline 80 & 0.0007204287034 & 0.0007275769085 & 0.000007148205148 & 0.00007746540234 & 0.00007940522370 & $1.939821358 * 10-6$ \\
\hline 90 & 0.0004044419029 & 0.0004084777420 & 0.000004035839088 & 0.00002441784774 & 0.00002502810988 & $6.102621357 * 10-7$ \\
\hline 100 & 0.0002270449703 & 0.0002293177469 & 0.000002272776554 & 0.000007695879186 & 0.000007887994360 & $1.921151739 * 10-7$ \\
\hline
\end{tabular}

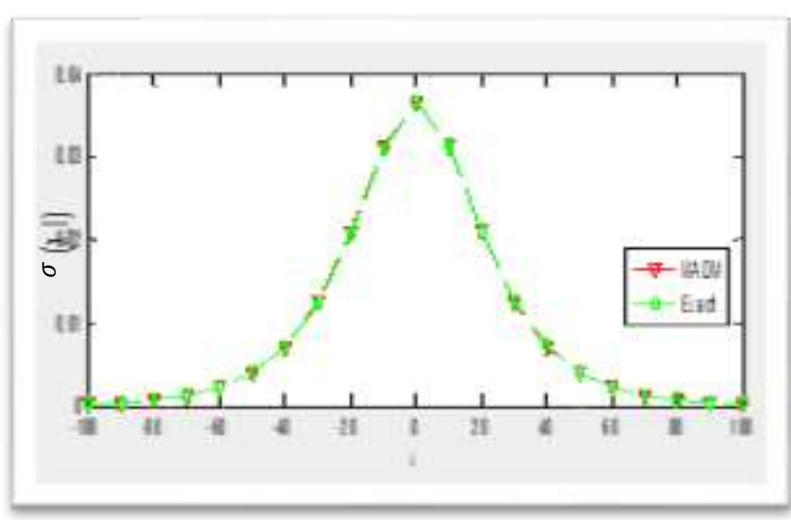

Figure 3. The MADM and Exact Solution $\sigma(\mathrm{x}, \mathrm{t})$ when $\mathrm{a}=0.1, \mathrm{t}=1, \mathrm{c}=0.01$

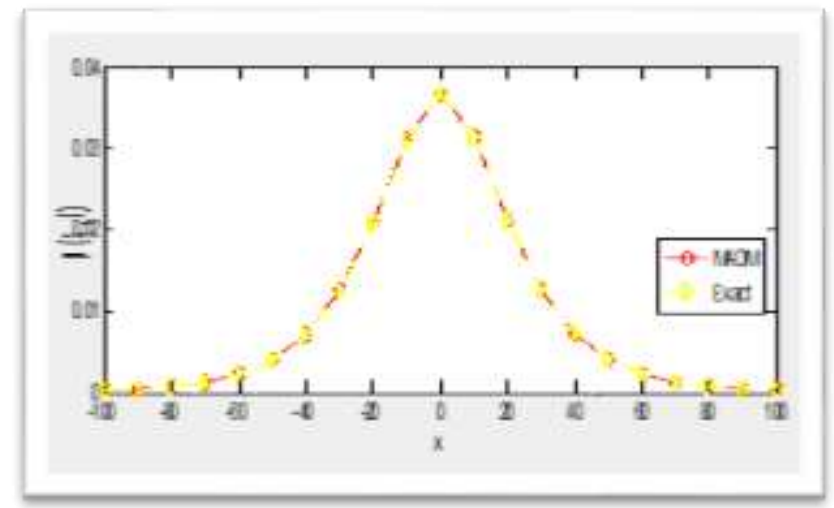

Figure 4. The MADM and Exact Solution $\mathrm{p}(\mathrm{x}, \mathrm{t})$ when $\mathrm{a}=0,1, \mathrm{t}=1, \mathrm{c}=0.01$

Table 3. (G_MADM) and exact solution when $\mathrm{a}=-10, \mathrm{c}=0.0001, \mathrm{t}=1$

\begin{tabular}{|c|c|c|c|c|c|c|}
\hline $\mathrm{x}$ & $\sigma_{\mathrm{G}_{-} \text {MADM }}$ & $\sigma_{\text {exact }}$ & $\left|\sigma_{\text {exact }}-\sigma_{\mathrm{G} \_ \text {MADM }}\right|$ & $\rho_{\mathrm{G} \_M A D M}$ & $\rho_{\text {exact }}$ & $\left|\rho_{\text {exact }}-\rho_{\mathrm{G} \_\mathrm{MADM}}\right|$ \\
\hline-100 & 0.00003117334540 & 0.00003117172202 & $1.623403661 * 10^{-9}$ & 0.00001457662036 & 0.00001457514381 & $1.476557610^{*} 10^{-9}$ \\
\hline-90 & 0.00003208501985 & 0.00003208348803 & $1.531843652 * 10^{-9}$ & 0.00001544168267 & 0.00001544025307 & $1.429607941 * 10^{-9}$ \\
\hline-80 & 0.00003293849112 & 0.00003293706933 & $1.421813135^{*} 10^{-9}$ & 0.00001627411621 & 0.00001627275804 & $1.358171087 * 10^{-9}$ \\
\hline-60 & 0.00003372309408 & 0.00003372180071 & $1.293392059 * 10^{-9}$ & 0.00001705865902 & 0.00001705739765 & $1.261375701 * 10^{-9}$ \\
\hline-70 & 0.00003442839369 & 0.00003442724645 & $1.147260393 * 10^{-9}$ & 0.00001777966879 & 0.00001777852948 & $1.139314644 * 10^{-9}$ \\
\hline-50 & 0.00003504448203 & 0.00003504349742 & $9.846281322 * 10^{-10}$ & 0.00001842169367 & 0.00001842070067 & $9.930094680 * 10^{-10}$ \\
\hline-40 & 0.00003556228720 & 0.00003556148008 & $8.071353057 * 10^{-10}$ & 0.00001897010739 & 0.00001896928298 & $8.244104918 * 10^{-10}$ \\
\hline-30 & 0.00003597387860 & 0.00003597326152 & $6.170919726^{*} 10^{-10}$ & 0.00001941176971 & 0.00001941113317 & $6.365480328 * 10^{-10}$ \\
\hline-20 & 0.00003627275003 & 0.00003627233286 & $4.171782260 * 10^{-10}$ & 0.00001973566538 & 0.00001973523197 & $4.334094799 * 10^{-10}$ \\
\hline-10 & 0.00003645406351 & 0.00003645385310 & $2.104141872 * 10^{-10}$ & 0.00001993347061 & $0.0000199332510 \mathrm{~S}$ & $2.195247862 * 10^{-10}$ \\
\hline 0 & 0.00003651483716 & 0.00003651483698 & $1.8 * 10^{-13}$ & 0.00001999999999 & $0.0000199999998 \mathrm{C}$ & $1.900000094 * 10^{-13}$ \\
\hline 10 & 0.00003645406351 & 0.00003645427357 & $2.100641941 * 10^{-10}$ & 0.00001993349175 & 0.00001993371092 & $2.191747877 * 10^{-10}$ \\
\hline 20 & 0.00003627275003 & 0.00003627316684 & $4.168182331 * 10^{-10}$ & 0.00001973570646 & 0.00001973613949 & $4.330294816 * 10^{-10}$ \\
\hline 30 & 0.00003597387860 & 0.00003597449537 & $6.167819787 * 10^{-10}$ & 0.00001941182851 & $0.0000194124647 €$ & $6.362580341 * 10^{-10}$ \\
\hline 40 & 0.00003556228720 & 0.00003556309403 & $8.068453112 * 10^{-10}$ & 0.00001897018077 & 0.00001897100486 & $8.240904932 * 10^{-10}$ \\
\hline 50 & 0.00003504448203 & 0.00003504546632 & $9.843081381 * 10^{-10}$ & 0.00001842177797 & 0.00001842277065 & $9.926894695 * 10^{-10}$ \\
\hline 60 & 0.00003442839369 & 0.00003442954068 & $1.147010397 * 10^{-9}$ & 0.00001777976001 & 0.00001778089907 & $1.139064645^{*} 10^{-9}$ \\
\hline 70 & 0.00003372309408 & 0.00003372438721 & $1.293152063 * 10^{-9}$ & 0.00001705875322 & 0.00001706001439 & $1.261175702 * 10^{-9}$ \\
\hline 80 & 0.00003293849112 & 0.00003293991268 & $1.421583138 * 10^{-9}$ & 0.00001627420971 & 0.00001627556772 & $1.358011088 * 10^{-9}$ \\
\hline 90 & 0.00003208501985 & 0.00003208655153 & $1.531703654 * 10^{-9}$ & 0.00001544177231 & 0.00001544320184 & $1.429537941 * 10^{-9}$ \\
\hline 100 & 0.00003117334540 & 0.00003117496865 & $1.623273663 * 10^{-9}$ & 0.00001457670356 & 0.00001457818005 & $1.476497610^{*} * 10^{-9}$ \\
\hline
\end{tabular}

Table 4. The absolute error between the real solution and MADM, G-MADM methods 


\begin{tabular}{|c|c|c|c|}
\hline $\begin{array}{c}\left|\sigma_{\text {exact }}-\sigma_{\text {MADM }}\right| \\
\mathrm{a}=\mathbf{0 . 1}, \boldsymbol{c}=\mathbf{0 . 0 0 0 1}, t=\mathbf{1}\end{array}$ & $\begin{array}{c}\left|\rho_{\text {exact }}-\rho_{\text {MADM }}\right| \\
a=0.1, c=0.0001, t=1\end{array}$ & $\begin{array}{c}\left|\sigma_{\text {exact }}-\sigma_{\mathrm{G}_{\mathrm{MADDM}}}\right| \\
a=-\mathbf{1 0}, \boldsymbol{c}=\mathbf{0 . 0 0 0 1}, t=1\end{array}$ & $\begin{array}{c}\left|\rho_{\text {exact }}-\rho_{\mathrm{G}_{\mathrm{GMADM}}}\right| \\
a=-10, c=0.0001, t=1\end{array}$ \\
\hline $1.535731237 * 10^{-8}$ & $1.517536499 * 10^{-8}$ & $1.623403661 * 10^{-9}$ & $1.476557610^{*} 10^{-9}$ \\
\hline $1.446696319 * 10^{-8}$ & $1.470569996 * 10^{-8}$ & $1.531843652 * 10^{-9}$ & $1.429607941 * 10^{-9}$ \\
\hline $1.217831272 * 10^{-8}$ & $1.299580908 * 10^{-8}$ & $1.293392059 * 10^{-9}$ & $1.261375701 * 10^{-9}$ \\
\hline $1.078835673 * 10^{-8}$ & $1.174634934 * 10^{-8}$ & $1.147260393 * 10^{-9}$ & $1.139314644 * 10^{-9}$ \\
\hline $9.248549282 * 10^{-9}$ & $1.024415783 * 10^{-8}$ & $9.846281322 * 10^{-10}$ & $9.930094680 * 10^{-10}$ \\
\hline $3.909620839 * 10^{-9}$ & $4.476611928 * 10^{-7}$ & $4.171782260 * 10^{-10}$ & $4.334094799 * 10^{-10}$ \\
\hline $1.971257584 * 10^{-9}$ & $2.267820866 * 10^{-7}$ & $2.104141872 * 10^{-10}$ & $2.195247862 * 10^{-10}$ \\
\hline $1.7 * 10^{-12}$ & $1.9 * 10^{-11}$ & $1.8 * 10^{-13}$ & $1.900000094 * 10^{-13}$ \\
\hline $1.967857584 * 10^{-9}$ & $2.264320866 * 10^{-8}$ & $2.100641941 * 10^{-10}$ & $2.191747877 * 10^{-10}$ \\
\hline $3.906120839 * 10^{-9}$ & $4.472811928 * 10^{-8}$ & $4.168182331 * 10^{-10}$ & $4.330294816^{*} 10^{-10}$ \\
\hline $1.217581272 * 10^{-8}$ & $1.299380908 * 10^{-7}$ & $1.293152063 * 10^{-9}$ & $1.261175702 * 10^{-9}$ \\
\hline $1.340451763 * 10^{-8}$ & $1.398078919 * 10^{-7}$ & $1.421583138 * 10^{-9}$ & $1.358011088 * 10^{-9}$ \\
\hline $1.446546319 * 10^{-8}$ & $1.470499996 * 10^{-7}$ & $1.531703654 * 10^{-9}$ & $1.429537941 * 10^{-9}$ \\
\hline $1.535591237 * 10^{-8}$ & $1.517476499 * 10^{-7}$ & $1.623273663 * 10^{-9}$ & $1.476497610 * 10^{-9}$ \\
\hline
\end{tabular}

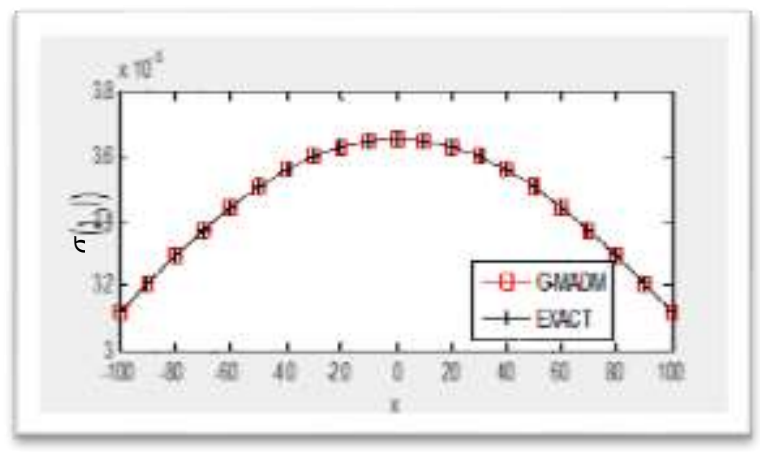

Figure 5. The G-MADM and Exact Solution $\sigma(x . t)$ when $a=-10 . t=1 . c=0.0001$

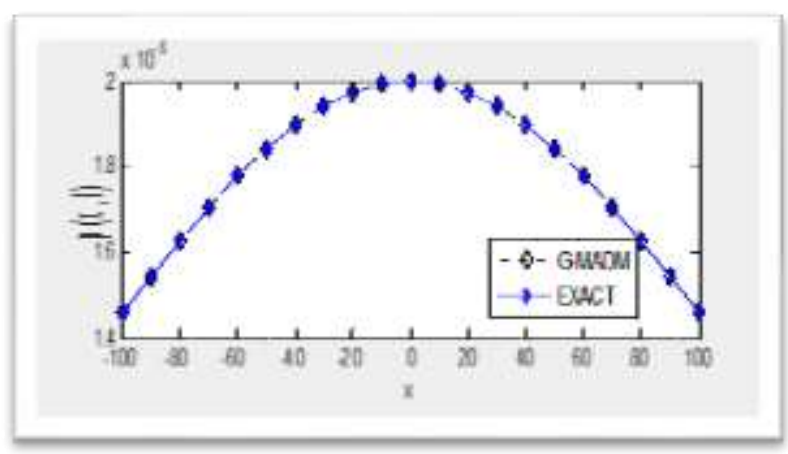

Figure 6. The G-MADM and Exact Solution $\mathrm{p}(\mathrm{x}, \mathrm{t})$ when $\mathrm{a}=-10, \mathrm{t}=1, \mathrm{c}=0.0001$

\section{CONCLUSIONS}

The DSW system was solved by using MADN. We noticed that this method is extremely fast in convergent to the real solution. Furthermore, the actual behaviour of the problem may be altered if the speed and convergence are not constrained by any restrictive assumptions. Also, the lower value of $\mathrm{C}$ and $\mathrm{T}$, which solution approaches the real solution. Genetic algorithm has also been used to find the optimum value of the parameter (a). The absolute error between the real solution and the G-MADM, MADM methods calculated. All calculations are conducted with the help of Maple 18 and Matlab Programs.

\section{ACKNOWLEDGEMENTS}

The authors are very grateful to the University of Mosul/College of Basic Education and the College of physical education and sport sciences for their provided facilities, which helped to improve the quality of this work.

\section{REFERENCES}

[1] A. Wazwaz, "The decomposition method applied to systems of partial differential equations and to the reactiondiffusion brusselator model," Applied Mathematics and Computation, vol. 110, no. 2-3, pp. 251-264, 2000, doi: 10.1016/s0096-3003(99)00131-9.

[2] A. Wazwaz, "A comparison between the variational iteration method and Adomian decomposition method," vol. 207, pp. 129-136, 2007, doi: 10.1016/j.cam.2006.07.018.

[3] M. El-Borai, A. A. Zaghrout, and A. M. Elshaer, "Exact solutions for nonlinear partial differential equations by using cosine-function method," vol. 9, no. 3, 2011. 
[4] G. Adomian, "Non-linear stochastic operator equations", Academic Press, San Diego, CA. 1986.

[5] N. A. Al-thanoon, O. S. Qasim, and Z. Y. Algamal, "Tuning parameter estimation in SCAD-support vector machine using firefly algorithm with application in gene selection and cancer classification," Comput. Biol. Med., 2018, doi: 10.1016/j.compbiomed.2018.10.034.

[6] He. Y. He, Y. Long, and L. Shaolin, "Exact Solutions of the Drinfel'd-Sokolov-Wilson Equation Using the F Expansion Method Combined with Exp-Function Method." International Mathematical, vol. 5, no. 65, pp. 3231$3242,2010$.

[7] Arora, Rajan and Anoop Kumar, "Solution of the Coupled Drinfeld's - Sokolov - Wilson (DSW) system by Homotopy Analysis Method," Adv Sci Engng, Med., vol. 5, no. 10, pp. 1105-1111, 2010. Doi: 10.1166/asem.2013.1399.

[8] A. H. Ali, "Adomian decomposition method for solving some models of nonlinear partial differential equations," Basrah Journal of Science, vol. 26, no. 1, pp. 1-11, 2008.

[9] R. S. Abdul-jabbar, "A numerical scheme for the solution of fractional integro- differential equations using the Adomian decomposition," Journal of university of Anbar for Pure science, vol. 6, no. 1, pp. 64-66, 2012.

[10] M. Ghoreishi, P. Pinang, and N. H. M. Ali, "Adomian Decomposition Method ( ADM ) for Nonlinear Wave-like Equations with Variable Coefficient 1 Introduction," Applied Mathematical Sciences, vol. 4, no. 49, pp. 2431-2444, 2010.

[11] J. Duan, T. Chaolu, R. Rach, and L. Lu, "The Adomian decomposition method with convergence acceleration techniques for nonlinear fractional differential equations," Comput. Math. with Appl., vol. 66, no. 5, pp. 728-736, 2013, doi: 10.1016/j.camwa.2013.01.019.

[12] W. Al-hayani, "An Efficient Advanced Adomian Decomposition Method to Solve Second-Order Boundary Value Problems with Neumann Conditions," International Mathematical Forum, vol. 10, no. 1, pp. 13-23, 2015, doi: 10.12988/IMF.2015.410182.

[13] O. Gonz, "Applying Adomian Decomposition Method to Solve Burgess Equation with a Non-Linear Source," Int. J. Appl. Comput. Math, vol. 3, pp. 213-224, 2017, doi: 10.1007/s40819-015-0100-4.

[14] A. F. Qasim and M. A. Hilal, "Solving a Class of High Order Non-linear Partial Differential Systems Using Adomian Decomposition Method Adomian,” Diyala Journal For Pure Science, vol. 14, no. 2, pp. 76-93, 2017, doi: 10.24237/DJPS.1402.378B.

[15] Wazwaz, Abdul-majid. "A reliable medication of adomian decomposition method" Applied Mathematics and Computation, vol. 102, no. 1, pp. 77-86. 1999, doi: 10.1016/S0096-3003(98)10024-3.

[16] X. Luo, "A two-step Adomian decomposition method," Applied Mathematics and Computation, vol. 170, no.1, pp. 570-583, 2005, doi: 10.1016/j.amc.2004.12.010.

[17] H. Jafari, E. Tayyebi, S. Sadeghi, and C. M. Khalique, "A new modification of the Adomian decomposition method for nonlinear integral equations MSC, " vol. 1, no. 4, pp. 33-39, 2014.

[18] M. Basto, V. Semiao, and F. L. Calheiros, "Numerical study of modified Adomian's method applied to Burgers equation," Journal of Computational and Applied Mathematics, vol. 206, pp. 927-949, 2007, doi: 10.1016/j.cam.2006.09.001.

[19] H. O. Bakodah, "Modified adomain decomposition method for the generalized fifth order KdV equations," American Journal of Computational Mathematics, no. January 2013, 2017, doi: 10.4236/ajcm.2013.31008.

[20] A. F. Qasim and Z. Y. Ali, "Application of Modified Adomian Decomposition Method to (2+1) - dimensional Nonlinear Wu-Zhang system," Journal of Al-Qadisiyah for Computer Science and Mathematics, vol. 10, no. 1, pp. 4053, 2018, doi: 10.29304/jqcm.2018.10.1.340.

[21] A. Wazwaz, "A comparison between the variational iteration method and Adomian decomposition method," Journal of Computational and Applied Mathematics, vol. 207, no.1, pp. 129-136, 2007, doi: 10.1016/j.cam.2006.07.018.

[22] Z. Ranen, "Homotopy perturbation method for solving the nonlinear wave equations." JNUS, vol. 13, no. 4, pp. 190-193, 2010. Doi: 10.22401/jnus.13.4.26.

[23] J. Ann and M. Al-Amr, "Numerical solution of a Reaction-Diffusion system with fast reversible reaction by using adomian's decomposition method and he's variational iteration method," AL-Rafidain Journal of Computer Sciences and Mathematics, vol. 9, no. 2, pp. 243-257, 2012, doi: 10.33899/csmj.2012.163715.

[24] Z. A. Firoozjae, "The comparison adomian decomposition method and differential quadrature method for solving some nonlinear partial diferential equations," American Journal of Applied Mathematics, vol. 3, no. 3, pp. 90-94, 2015, doi: 10.11648/j.ajam.20150303.12.

[25] G. Adomian, "Solution of Physical Problems by decomposition," Appl. Math. Comput., vol. 27, no. 9-10, pp. 145154, 1994, doi: 10.1016/0898-1221(94)90132-5.

[26] El-Kalla, I. L., "Error Analysis of Adomian Series Solution to A Class of Nonlinear Differential Equations", Applied Mathematics E-Notes, vol. 7, pp. 214-221, 2007.

[27] T. Y. Wu, J.E. Zhang, "On modelling nonlinear long wave", in: L.P. Cook, V. Roytbhurd, M. Tulin (Eds.), Mathematics Is for Solving Problems, Society for Industrial and Applied Mathematics, Philadelphia, 1996, pp. 233. 


\section{BIOGRAPHIES OF AUTHORS}
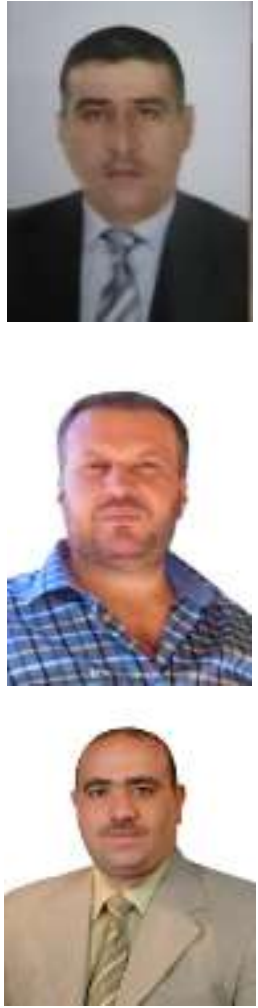

Badran Jasim Salim received his Ph.D In Mathematics, 2015, College of Mathematics, University of Voronezh/Russia. M.Sc In Mathematics, 2004, Mathematics Department, College of Computer Science and Mathematics, University of Mosul/Iraq. Lecture/Full-Time Lecturer at Dept. of Mathematics/Faculty of Basic education/University of Mosul/Iraq 2006. My research interest includes Numerical Modeling, Numerical Analysis, Optimization.

Oday Ahmed Jasim received his Master degrees in Apply Mathematics science from the College of Computer Science and Mathematics, University of Mosul, Iraq, in 2013. Since 2013, I am currently a lecturer of Mathematics science in the Department of Mathematics, College of Basic Education at the University of Mosul, Iraq. My research interest includes Numerical Modeling, Numerical Analysis, Optimization.

Zeiad Yahya Ali received a master's degree in applied mathematics from the College of Education/Department of Mathematics, University of Mosul, Iraq, in 2010. Since 2010, I am currently working as a lecturer in the Sports Sciences Branch, College of Physical Education and Sports Sciences at the University of Mosul, Iraq. My research interests include numerical modelling, numerical analysis, optimization, and statistics. 